TRANSACTIONS OF THE

AMERICAN MATHEMATICAL SOCIETY

Volume 364, Number 5, May 2012, Pages 2377-2391

S 0002-9947(2011)05446-2

Article electronically published on December 13, 2011

\title{
ON LOCALLY CONFORMALLY FLAT GRADIENT STEADY RICCI SOLITONS
}

\author{
HUAI-DONG CAO AND QIANG CHEN
}

\begin{abstract}
In this paper, we classify $n$-dimensional $(n \geq 3)$ complete noncompact locally conformally flat gradient steady solitons. In particular, we prove that a complete noncompact nonflat locally conformally flat gradient steady Ricci soliton is, up to scaling, the Bryant soliton.
\end{abstract}

\section{The RESUlts}

A complete Riemannian metric $g_{i j}$ on a smooth manifold $M^{n}$ is called a gradient steady Ricci soliton if there exists a smooth function $F$ on $M^{n}$ such that the Ricci tensor $R_{i j}$ of the metric $g_{i j}$ is given by the Hessian of $F$ :

$$
R_{i j}=\nabla_{i} \nabla_{j} F \text {. }
$$

The function $F$ is called a potential function of the gradient steady soliton. Clearly, when $F$ is a constant the gradient steady Ricci soliton is simply a Ricci flat manifold. Thus Ricci solitons are natural extensions of Einstein metrics. Gradient steady solitons play an important role in Hamilton's Ricci flow as they correspond to translating solutions, and often arise as Type II singularity models. Thus one is interested in classifying them and understanding their geometry.

It turns out that compact gradient steady solitons must be Ricci flat. In dimension $n=2$, Hamilton [14] discovered the first example of a complete noncompact gradient steady soliton on $\mathbb{R}^{2}$, called the cigar soliton, where the metric is given by

$$
d s^{2}=\frac{d x^{2}+d y^{2}}{1+x^{2}+y^{2}} .
$$

The cigar soliton has positive curvature and is asymptotic to a cylinder of finite circumference at infinity. Furthermore, Hamilton 14 showed that the only complete steady soliton on a two-dimensional manifold with bounded (scalar) curvature $R$ which assumes its maximum at an origin is, up to scaling, the cigar soliton. For $n \geq 3$, Bryant 2 proved that there exists, up to scaling, a unique complete rotationally symmetric gradient Ricci soliton on $\mathbb{R}^{n}$, see, e.g., Chow et al. 8 for details. The Bryant soliton has positive sectional curvature, linear curvature decay and volume growth on the order of $r^{(n+1) / 2}$. In the Kähler case, the first author [3] constructed a complete gradient steady Kähler-Ricci soliton on $\mathbb{C}^{m}$, for $m \geq 2$, with positive sectional curvature and $U(m)$ symmetry.

Received by the editors March 9, 2010 and, in revised form, March 15, 2010.

2010 Mathematics Subject Classification. Primary 53C21, 53C25.

The first author was partially supported by NSF Grants DMS-0506084 and DMS-0909581.

The second author was partially supported by NSF Grant DMS-0354621 and a Dean's Fellowship of the School of Arts and Sciences at Lehigh University.

(C)2011 American Mathematical Society Reverts to public domain 28 years from publication 
A well-known conjecture is that, in dimension $n=3$, the Bryant soliton is the only complete noncompact ( $\kappa$-noncollapsed) gradient steady soliton with positive sectional curvature 1 We remark that S.-C. Chu [9] and H. Guo [12] have studied the geometry of 3-dimensional gradient steady solitons with positive sectional curvature and the scalar curvature $R$ attaining its maximum at some origin. For $n \geq 4$, it is also natural to ask if the Bryant soliton is the only complete noncompact, positively curved, locally conformally flat gradient steady soliton. In this paper, we classify $n$ dimensional $(n \geq 3)$ complete noncompact locally conformally flat gradient steady solitons and give an affirmative answer to the latter question. Our main results:

Theorem 1.1. Let $\left(M^{n}, g_{i j}, F\right), n \geq 3$, be an $n$-dimensional complete noncompact locally conformally flat gradient steady Ricci soliton with positive sectional curvature. Then, $\left(M^{n}, g_{i j}, F\right)$ is isometric to the Bryant soliton.

Theorem 1.2. Let $\left(M^{n}, g_{i j}, F\right), n \geq 3$, be an $n$-dimensional complete noncompact locally conformally flat gradient steady Ricci soliton. Then, $\left(M^{n}, g_{i j}, F\right)$ is either flat or isometric to the Bryant soliton.

Our work was motivated in part by the works of physicists Israel [16] and Robinson 21 concerning the uniqueness of the Schwarzschild black hole among all static, asymptotically flat vacuum space-times. In their setting, the Einstein field equations take the form

$$
R_{i j}+V^{-1} \nabla_{i} \nabla_{j} V=0
$$

and

$$
\Delta V=0
$$

for a certain positive potential function $V$ on a three-dimensional space-like hypersurface $\left(N^{3}, g_{i j}\right)$. They proved that such $\left(N^{3}, g_{i j}, V\right)$ must be rotationally symmetric without assuming locally conformal flatness. In fact, they were able to prove that such $\left(N^{3}, g_{i j}, V\right)$ is locally conformally flat. However, it remains a challenge to do the same for 3-dimensional gradient steady Ricci solitons.

\section{Preliminaries}

In this section, we recall some basic facts and collect several known results about gradient steady solitons.

Let $\left(M^{n}, g_{i j}, F\right)$ be a gradient steady Ricci soliton so that the Ricci tensor $R_{i j}$ of the metric $g_{i j}$ is given by the Hessian of the potential function $F$ :

$$
R_{i j}=\nabla_{i} \nabla_{j} F \text {. }
$$

Taking the covariant derivatives and using the commutating formula for covariant derivatives, we obtain

$$
\nabla_{i} R_{j k}-\nabla_{j} R_{i k}=R_{i j k l} \nabla_{l} F .
$$

Taking the trace on $j$ and $k$, and using the contracted second Bianchi identity

$$
\nabla_{j} R_{i j}=\frac{1}{2} \nabla_{i} R
$$

we get

$$
\nabla_{i} R=-2 R_{i j} \nabla_{j} F
$$

\footnotetext{
${ }^{1}$ Perelman $([20], 11.9)$ claimed that the conjecture is true but didn't give any detail, or sketch, of a proof.
} 
Thus

$$
\nabla_{i}\left(R+|\nabla F|^{2}\right)=-2\left(R_{i j}-\nabla_{i} \nabla_{j} F\right) \nabla F=0
$$

Hence we have

Lemma 2.1 (Hamilton [15]). Let $\left(M^{n}, g_{i j}, F\right)$ be a complete gradient steady soliton satisfying (1.1). Then we have

$$
\nabla_{i} R=-2 R_{i j} \nabla_{j} F
$$

and

$$
R+|\nabla F|^{2}=C_{0}
$$

for some constant $C_{0}$. Here $R$ denotes the scalar curvature.

Taking the trace in (1.1), we get

$$
R=\Delta F .
$$

Combining (2.4) with (2.3), we have

$$
\Delta F+|\nabla F|^{2}=C_{0}
$$

If $M$ is compact, then it follows that

$$
0=\int_{M} \Delta\left(e^{F}\right)=\int_{M}\left(\Delta F+|\nabla F|^{2}\right) e^{F}=C_{0} \int_{M} e^{F} .
$$

Thus $C_{0}=0$. Now integrating (2.5), with $C_{0}=0$, over $M$ yields

$$
\int_{M}|\nabla F|^{2}=0
$$

implying that $F$ is a constant. Therefore, we get

Proposition 2.1 (cf. Hamilton [15], Ivey [17]). On a compact manifold $M^{n}$, a gradient steady Ricci soliton is necessarily a Ricci flat Einstein metric.

Complete noncompact steady solitons do exist and here are some basic examples:

Example 2.1 (The cigar soliton). In dimension two, Hamilton 14 discovered the first example of a complete noncompact steady soliton on $\mathbb{R}^{2}$, called the cigar soliton, where the metric is given by

$$
d s^{2}=\frac{d x^{2}+d y^{2}}{1+x^{2}+y^{2}}
$$

with potential function

$$
F=\log \left(1+x^{2}+y^{2}\right)
$$

The (scalar) curvature of the cigar soliton is given by

$$
R=\frac{1}{1+x^{2}+y^{2}} .
$$

Hence it is positive, attains its maximum at the origin, and decays to zero exponentially fast (in terms of the geodesic distance) at space infinity. Furthermore, the cigar soliton has linear volume growth and is asymptotic to a cylinder of finite circumference at $\infty$. 
Example 2.2 (The Bryant soliton). In the Riemannian case, higher-dimensional examples of noncompact gradient steady solitons were found by Robert Bryant [2] on $\mathbb{R}^{n}(n \geq 3)$. They are rotationally symmetric and have positive sectional curvature. Furthermore, the geodesic sphere $S^{n-1}$ of radius $s$ has a diameter of the order $\sqrt{s}$. Thus the volume of the geodesic balls $B_{r}(0)$ grows according to the order of $r^{(n+1) / 2}$.

Example 2.3 (Noncompact gradient steady Kähler solitons). In the Kähler case, the first author [3] found two examples of complete rotationally symmetric noncompact gradient steady Kähler-Ricci solitons

(a) On $\mathbb{C}^{n}$ (for $n=1$ it is just the cigar soliton). These examples are $U(n)$ invariant and have positive sectional curvature. It is interesting to point out that the geodesic sphere $S^{2 n-1}$ of radius $s$ is an $S^{1}$-bundle over $\mathbb{C} P^{n-1}$ where the diameter of $S^{1}$ is of the order 1 , while the diameter of $\mathbb{C} P^{n-1}$ is of the order $\sqrt{s}$. Thus the volume of the geodesic balls $B_{r}(0)$ grows according to the order of $r^{n}, n$ being the complex dimension. Also, the curvature $R(x)$ decays like $1 / r$.

(b) On the blowup of $\mathbb{C}^{n} / \mathbb{Z}_{n}$ at the origin. This is the same space on which Eguchi-Hansen $(n=2)$ and Calabi $(n \geq 2)$ constructed examples of hyper-Kähler metrics. For $n=2$, the underlying space is the canonical line bundle over $\mathbb{C P}^{1}$.

Now a Ricci flat metric is clearly a stationary solution of Hamilton's Ricci flow

$$
\frac{\partial g_{i j}(t)}{\partial t}=-2 R_{i j}(t)
$$

This happens, for example, on a flat torus or on any $K 3$-surface with a Calabi-Yau metric.

On the other hand, suppose that we have a complete steady Ricci soliton $g_{i j}$ on a smooth manifold $M^{n}$ with potential function $F$. As observed by Z.-H. Zhang 22], the gradient vector field $V=\nabla F$ is a complete vector field on $M$. Let $\varphi_{t}$ denote the one-parameter group of diffeomorphisms of $M^{n}$ generated by $-V$. Then it easily follows that

$$
\tilde{g}_{i j}(t)=\varphi_{t}^{*} g_{i j}
$$

is a solution to the Ricci flow for $-\infty<t<\infty$, with $\tilde{g}_{i j}(0)=g_{i j}$.

Next we present a useful result, which was implicitly proved by B.-L. Chen 7 . For the reader's convenience, we include a proof here (see also Proposition 5.5 in (4).

Proposition 2.2. Let $g_{i j}(t)$ be a complete ancient solution to the Ricci flow on a noncompact manifold $M^{n}$. Then the scalar curvature $R$ of $g_{i j}(t)$ is nonnegative for all $t$.

Proof. Suppose $g_{i j}(t)$ is defined for $-\infty<t \leq T$ for some $T>0$. We divide the argument into two steps:

Step 1 . Consider any complete solution $g_{i j}(t)$ defined on $[0, T]$. For any fixed point $x_{0} \in M$, pick $r_{0}>0$ sufficiently small so that

$$
|R c|(\cdot, t) \leq(n-1) r_{0}^{-2} \quad \text { on } B_{t}\left(x_{0}, r_{0}\right)
$$

for all $t \in[0, T]$. Then for any positive number $A>2$, pick $K_{A}>0$ such that $R \geq-K_{A}$ on $B_{0}\left(x_{0}, A r_{0}\right)$ at $t=0$. We claim that there exists a universal constant 
$C>0$ (depending on the dimension $n$ ) such that

$$
R(\cdot, t) \geq \min \left\{-\frac{n}{t+\frac{1}{K_{A}}},-\frac{C}{A r_{0}^{2}}\right\} \quad \text { on } B_{t}\left(x_{0}, \frac{3 A}{4} r_{0}\right)
$$

for each $t \in[0, T]$.

Indeed, take a smooth nonnegative decreasing function $\phi$ on $\mathbb{R}$ such that $\phi=1$ on $(-\infty, 7 / 8]$, and $\phi=0$ on $[1, \infty)$. Consider the function

$$
u(x, t)=\phi\left(\frac{d_{t}\left(x_{0}, x\right)}{A r_{0}}\right) R(x, t),
$$

where $d_{t}\left(x_{0}, x\right)$ is the distance function from $x_{0}$ to $x$ at time $t$. Then we have

$$
\left(\frac{\partial}{\partial t}-\Delta\right) u=\frac{\phi^{\prime} R}{A r_{0}}\left(\frac{\partial}{\partial t}-\Delta\right) d_{t}\left(x_{0}, x\right)-\frac{\phi^{\prime \prime} R}{\left(A r_{0}\right)^{2}}+2 \phi|R c|^{2}-2 \nabla \phi \cdot \nabla R
$$

at smooth points of $d_{t}\left(x_{0}, \cdot\right)$.

Let $u_{\min }(t)=\min _{M} u(\cdot, t)$. Whenever $u_{\min }\left(t_{0}\right) \leq 0$, assume $u_{\min }\left(t_{0}\right)$ is achieved at some point $\bar{x} \in B_{t_{0}}\left(x_{0}, r_{0}\right)$; then $\phi^{\prime} R\left(\bar{x}, t_{0}\right) \geq 0$. On the other hand, by Lemma 8.3(a) of Perelman [20] (see also Lemma 3.4.1 (i) of [5]), we know that

$$
\left(\frac{\partial}{\partial t}-\Delta\right) d_{t}\left(x_{0}, x\right) \geq-\frac{5(n-1)}{3 r_{0}}
$$

outside $B_{t}\left(x_{0}, r_{0}\right)$. Following (Section 3, Hamilton [13]), we define

$$
\left.\frac{d}{d t}\right|_{t=t_{0}} u_{\min }=\liminf _{h \rightarrow 0^{+}} \frac{u_{\min }\left(t_{0}+h\right)-u_{\min }\left(t_{0}\right)}{h},
$$

the liminf of all forward difference quotients. Then

$$
\left.\frac{d}{d t}\right|_{t=t_{0}} u_{\min } \geq-\frac{5(n-1)}{3 A r_{0}^{2}} \phi^{\prime} R+\frac{2}{n} \phi R^{2}+\frac{1}{\left(A r_{0}\right)^{2}}\left(\frac{2 \phi^{\prime 2}}{\phi}-\phi^{\prime \prime}\right) R .
$$

Hence,

$$
\left.\frac{d}{d t}\right|_{t=t_{0}} u_{\min } \geq \frac{1}{n} u_{\min }^{2}\left(t_{0}\right)-\frac{C^{2}}{\left(A r_{0}^{2}\right)^{2}}
$$

provided $u_{\min }\left(t_{0}\right) \leq 0$. Now integrating the above inequality, we get

$$
u_{\min }(t) \geq \min \left\{-\frac{n}{t+\frac{1}{K_{A}}},-\frac{C}{A r_{0}^{2}}\right\} \quad \text { on } B_{t}\left(x_{0}, \frac{3 A}{4} r_{0}\right),
$$

and the inequality (2.6) in our claim follows.

Step 2. Now if our solution $g_{i j}(t)$ is ancient, we can replace $t$ by $t-\alpha$ in (2.6) and get

$$
R(\cdot, t) \geq \min \left\{-\frac{n}{t-\alpha+\frac{1}{K_{A}}},-\frac{C}{A r_{0}^{2}}\right\} \quad \text { on } B_{t}\left(x_{0}, \frac{3 A}{4} r_{0}\right) .
$$

Letting $A \rightarrow \infty$ and then $\alpha \rightarrow-\infty$, we see that $R(\cdot, t) \geq 0$ for all $t$. This completes the proof of Proposition 2.2.

As an immediate corollary, we have

Lemma 2.2. Let $\left(M^{n}, g_{i j}, F\right)$ be a complete gradient steady soliton. Then it has nonnegative scalar curvature $R \geq 0$. 
We remark that Munteanu-Sesum [19] recently proved a Liouville-type theorem for gradient steady solitons; namely, a gradient steady soliton does not admit any nontrivial harmonic function with finite Dirichlet energy. As a consequence, a gradient steady soliton has at most one nonparabolic end.

For steady solitons with nonnegative Ricci curvature, we have the following.

Lemma 2.3 (Hamilton [15]). Let $\left(M^{n}, g_{i j}, F\right)$ be a complete gradient steady soliton with nonnegative Ricci curvature $R c \geq 0$ and assume the scalar curvature $R$ attains its maximum at some point $x_{0}$. Then the potential function $F$ is weakly convex and attains its minimum at $x_{0}$.

Moreover, if the Ricci curvature of $\left(M^{n}, g_{i j}, F\right)$ is assumed to be positive, then Lemma 2.3 can be strengthened to the following 2

Proposition 2.3. Let $\left(M^{n}, g_{i j}, F\right)$ be a complete noncompact gradient steady soliton with positive Ricci curvature $R c>0$. Assume the scalar curvature $R$ attains its maximum at some origin $x_{0}$. Then, there exist some constants $0<c_{1} \leq \sqrt{C_{0}}$ and $c_{2}>0$ such that the potential function $F$ satisfies the estimates

$$
c_{1} r(x)-c_{2} \leq F(x) \leq \sqrt{C_{0}} r(x)+\left|F\left(x_{0}\right)\right|,
$$

where $r(x)=d\left(x_{0}, x\right)$ is the distance function from $x_{0}$, and $C_{0}=R_{\max }$ is the constant in (2.2). In particular, $F$ is a strictly convex exhaustion function achieving its minimum at the only critical point $x_{0}$, and the underlying manifold $M^{n}$ is diffeomorphic to $\mathbb{R}^{n}$.

Proof. It is clear that the upper estimate in (2.7) in fact holds for complete gradient steady solitons in general, because $|\nabla F|^{2} \leq C_{0}$ by (2.3) and Lemma 2.2.

To prove the lower estimate, we consider any minimizing normal geodesic $\gamma(s)$, $0 \leq s \leq s_{0}$ for large $s_{0}>0$, starting from the origin $x_{0}=\gamma(0)$. Denote by $X(s)=\dot{\gamma}(s)$ the unit tangent vector along $\gamma$, and $\dot{F}=\nabla_{X} F(\gamma(s))$. By (1.1), we have

$$
\nabla_{X} \dot{F}=\nabla_{X} \nabla_{X} F=R c(X, X) .
$$

Integrating (2.8) along $\gamma$ and noting that $x_{0}$ is the (unique) minimum point of $F$, we get, for $s \geq 1$,

$$
\dot{F}(\gamma(s))=\int_{0}^{s} R c(X, X) d s \geq \int_{0}^{1} R c(X, X) d s \geq c_{1},
$$

where $c_{1}>0$ is the least eigenvalue of $R c$ on the unit geodesic ball $B_{x_{0}}(1)$. Thus,

$$
F\left(\gamma\left(s_{0}\right)\right)=\int_{1}^{s_{0}} \dot{F}(\gamma(s)) d s+F(\gamma(1)) \geq c_{1} s_{0}-c_{1}+F(\gamma(1)) .
$$

Now we turn our attention to locally conformally flat steady Ricci solitons. For any Riemannian manifold $\left(M^{n}, g\right)$, let

$$
\begin{aligned}
W_{i j k l}= & R_{i j k l}-\frac{1}{n-2}\left(g_{i k} R_{j l}-g_{i l} R_{j k}-g_{j k} R_{i l}+g_{j l} R_{i k}\right) \\
& +\frac{R}{(n-1)(n-2)}\left(g_{i k} g_{j l}-g_{i l} g_{j k}\right)
\end{aligned}
$$

${ }^{2}$ See also Remark 5.5 in [4], as well as Lemma 3.2 in 9 . 
be the Weyl tensor, and denote by

$$
C_{i j k}=\nabla_{k} R_{i j}-\nabla_{j} R_{i k}-\frac{1}{2(n-1)}\left(g_{i j} \nabla_{k} R-g_{i k} \nabla_{j} R\right)
$$

the Cotton tensor. It is well known that, for $n=3, W_{i j k l}$ vanishes identically while $C_{i j k}=0$ if and only if $\left(M^{3}, g_{i j}\right)$ is locally conformally flat; for $n \geq 4, W_{i j k l}=0$ if and only if $\left(M^{n}, g_{i j}\right)$ is locally conformally flat. Moreover, for $n \geq 4$, the vanishing of the Weyl tensor $W_{i j k l}$ implies the vanishing of the Cotton tensor $C_{i j k}$, while $C_{i j k}=0$ corresponds to the Weyl tensor being harmonic.

In proving Theorem 1.2, we need the following important facts due to B.-L. Chen [7] (for $n=3$ ) and Z.-H. Zhang [23] (for $n \geq 4$ ):

Proposition 2.4. Let $\left(M^{n}, g_{i j}, F\right)$ be a complete gradient steady Ricci soliton. Then $\left(M^{n}, g_{i j}, F\right)$ has nonnegative curvature operator $R m \geq 0$, provided either

(a) $n=3$, or

(b) $n \geq 4$ and $\left(M^{n}, g_{i j}, F\right)$ is locally conformally flat.

Remark 2.1. Part (a) is a special case of a more general result due to B.-L. Chen [7] for 3-dimensional complete ancient solutions. Part (b) was essentially proved by Z.-H. Zhang 23. In fact, the same arguments in 23. imply that a complete ancient solution $g_{i j}(t)$ to the Ricci flow with vanishing Weyl tensor for each time $t$ is necessarily of nonnegative curvature operator.

Combining Proposition 2.4 with (2.3) in Lemma 2.1, we have

Proposition 2.5. Let $\left(M^{n}, g_{i j}, F\right)$ be a complete gradient steady soliton such that either $n=3$, or $n \geq 4$ and $\left(M^{n}, g_{i j}, F\right)$ is locally conformally flat. Then $\left(M^{n}, g_{i j}, F\right)$ has bounded and nonnegative curvature operator $0 \leq R m \leq C$.

\section{The proofs of Theorem 1.1 and Theorem 1.2}

Throughout this section, we assume that $\left(M^{n}, g_{i j}, F\right)(n \geq 3)$ is a complete noncompact locally conformally flat gradient steady soliton. We are going to prove Theorem 1.1, which is the same as Proposition 3.1 below, and Theorem 1.2.

Proposition 3.1. If $\left(M^{n}, g_{i j}, F\right), n \geq 3$, is an $n$-dimensional complete noncompact locally conformally flat gradient steady soliton with positive sectional curvature, then $\left(M^{n}, g_{i j}, F\right)$ is a rotationally symmetric gradient steady soliton on $\mathbb{R}^{n}$, hence isometric to the Bryant soliton.

Proof. First of all, since the sectional curvature of $\left(M^{n}, g_{i j}, F\right)$ is positive we know that $M^{n}$ is diffeomorphic to $\mathbb{R}^{n}$ by Gromoll-Meyer [11] (or by Proposition 2.3 if $R$ attains its maximum at some origin $x_{0}$ ). Moreover, since the Ricci curvature is positive, the potential function $F$ is strictly convex, thus having at most one critical point. Secondly, if we denote by $G=|\nabla F|^{2}$, then in any neighborhood, where $G \neq 0$, of the equipotential hypersurface

$$
\Sigma_{c}=:\{x \in M: F(x)=c\}
$$

of a regular value $c$ of $F$, we can express the metric $d s^{2}=g_{i j}(x) d x^{i} d x^{j}$ as

$$
d s^{2}=\frac{1}{G(F, \theta)} d F^{2}+g_{a b}(F, \theta) d \theta^{a} d \theta^{b},
$$


where $\theta=\left(\theta^{2}, \cdots, \theta^{n}\right)$ denotes intrinsic coordinates for $\Sigma_{c}$. It is clear that the key step in proving Proposition 3.1 is to show that in (3.1) we have $G=G(F)$, $g_{a b}=g_{a b}(F)$, and that $\left(\Sigma_{c}, g_{a b}\right)$ is a space form of positive curvature.

Note that an $n$-dimensional rotationally symmetric metric is of the form

$$
d s^{2}=\psi^{2}(t) d t^{2}+\varphi^{2}(t) \bar{g}
$$

where $\bar{g}$ is the standard metric on the unit sphere $\mathbb{S}^{n-1}$, and the Ricci tensor of such a metric is given by

$$
R c=(n-1)\left(-\frac{\varphi_{t t}}{\varphi}+\frac{\varphi_{t} \psi_{t}}{\varphi \psi}\right) d t^{2}-\left(\frac{\varphi \varphi_{t t}}{\psi^{2}}+\frac{(n-2) \varphi_{t}^{2}}{\psi^{2}}-\frac{\varphi \varphi_{t} \psi_{t}}{\psi^{3}}+2-n\right) \bar{g} .
$$

In particular, it has at most two distinct eigenvalues depending only on $t$.

We shall first derive a useful formula for the norm square of the Cotton tensor for steady solitons with vanishing Weyl tensor. This formula plays an important role in our derivation of the desired property of the Ricci tensor for the steady soliton metric. Moreover, as $W_{i j k l}=0$ always when $n=3$, this formula is of particular interest in the three-dimensional case.

Lemma 3.1. For any $n$-dimensional gradient steady soliton $(n \geq 3)$ with vanishing Weyl tensor $W_{i j k l}=0$, we have

$$
\left|C_{i j k}\right|^{2}=\frac{1}{(n-2)^{2}}\left(\left|R_{i k} \nabla_{j} F-R_{i j} \nabla_{k} F\right|^{2}-\frac{2}{(n-1)}\left|R \nabla F-\frac{1}{2} \nabla G\right|^{2}\right) .
$$

Here $C_{i j k}$ is the Cotton tensor defined by (2.9).

Proof. Notice that, by (1.1) and Lemma 2.1, we have

$$
\nabla G=2 R c(\nabla F, \cdot)=-\nabla R .
$$

Using (2.1), (3.3), and the assumption $W_{i j k l}=0$, we can express

$$
\begin{aligned}
C_{i j k}= & \nabla_{k} R_{i j}-\nabla_{j} R_{i k}-\frac{1}{2(n-1)}\left(g_{i j} \nabla_{k} R-g_{i k} \nabla_{j} R\right) \\
= & R_{k j i l} \nabla_{l} F+\frac{1}{2(n-1)}\left(g_{i j} \nabla_{k} G-g_{i k} \nabla_{j} G\right) \\
= & \frac{1}{n-2}\left(R_{i k} \nabla_{j} F-R_{i j} \nabla_{k} F\right)+\frac{1}{2(n-1)(n-2)}\left(g_{i k} \nabla_{j} G-g_{i j} \nabla_{k} G\right) \\
& -\frac{R}{(n-1)(n-2)}\left(g_{i k} \nabla_{j} F-g_{i j} \nabla_{k} F\right) .
\end{aligned}
$$

Hence, by direct computations, we have

$$
\begin{aligned}
\left|C_{i j k}\right|^{2}= & \frac{1}{(n-2)^{2}}\left|R_{i k} \nabla_{j} F-R_{i j} \nabla_{k} F\right|^{2}+\frac{1}{2(n-1)(n-2)^{2}}|\nabla G|^{2} \\
& +\frac{2 R^{2}}{(n-1)(n-2)^{2}}|\nabla F|^{2}+\frac{2}{(n-1)(n-2)^{2}}[R \nabla G \cdot \nabla F-R c(\nabla F, \nabla G)] \\
& -\frac{4 R}{(n-1)(n-2)^{2}}\left[R|\nabla F|^{2}-R c(\nabla F, \nabla F)\right]-\frac{2 R}{(n-1)(n-2)^{2}} \nabla G \cdot \nabla F .
\end{aligned}
$$

On the other hand, by (3.3),

$$
R c(\nabla F, \nabla G)=\frac{1}{2}|\nabla G|^{2}
$$


and

$$
R c(\nabla F, \nabla F)=\frac{1}{2} \nabla G \cdot \nabla F
$$

Thus,

$$
\begin{aligned}
\left|C_{i j k}\right|^{2}= & \frac{1}{(n-2)^{2}}\left|R_{i k} \nabla_{j} F-R_{i j} \nabla_{k} F\right|^{2} \\
& -\frac{1}{2(n-1)(n-2)^{2}}\left(|\nabla G|^{2}-4 R \nabla G \cdot \nabla F+4 R^{2}|\nabla F|^{2}\right) \\
= & \frac{1}{(n-2)^{2}}\left(\left|R_{i k} \nabla_{j} F-R_{i j} \nabla_{k} F\right|^{2}-\frac{2}{(n-1)}\left|R \nabla F-\frac{1}{2} \nabla G\right|^{2}\right) .
\end{aligned}
$$

Next, using Lemma 3.1, we show that the Ricci tensor of our $\left(M^{n}, g_{i j}, F\right)$ has, at least pointwisely, the desired property that it has at most two distinct eigenvalues.

Lemma 3.2. At any point $p \in \Sigma_{c}$, the Ricci tensor of $\left(M^{n}, g_{i j}, F\right)$ either has a unique eigenvalue $\lambda$, or has two distinct eigenvalues $\lambda$ and $\mu$ of multiplicity 1 and $n-1$ respectively. In either case, $e_{1}=\nabla F /|\nabla F|$ is an eigenvector with eigenvalue $\lambda$. In other words, for any orthonormal basis $e_{2}, \cdots, e_{n}$ tangent to the level surface $\Sigma_{c}$ at $p$, the Ricci tensor has the following properties:

(i) $R c\left(e_{1}, e_{1}\right)=R_{11}$,

(ii) $R c\left(e_{1}, e_{b}\right)=R_{1 b}=0, b=2, \cdots, n$,

(iii) $R c\left(e_{a}, e_{b}\right)=R_{a a} \delta_{a b}, a, b=2, \cdots, n$, where either $R_{11}=\cdots=R_{n n}=\lambda$ or $R_{11}=\lambda$ and $R_{22}=\cdots=R_{n n}=\mu$.

Proof. For any regular value $c$ of $F$, pick an orthonormal basis $\left\{E_{1}, \cdots, E_{n}\right\}$ of the tangent space $T_{p} M$ at $p \in \Sigma_{c}=\{x \in M: F(x)=c\}$ so that $R c\left(E_{i}, E_{j}\right)=\lambda_{i} \delta_{i j}$. Then, we have

$$
\left|R_{i k} \nabla_{j} F-R_{i j} \nabla_{k} F\right|^{2}=2 \sum_{j=1}^{n}\left|\nabla_{j} F\right|^{2} \sum_{i \neq j} \lambda_{i}^{2}
$$

and

$$
\left|R \nabla F-\frac{1}{2} \nabla G\right|^{2}=\sum_{j=1}^{n}\left|\nabla_{j} F\right|^{2}\left(\sum_{i \neq j} \lambda_{i}\right)^{2} .
$$

Plugging the above two identities into (3.2) and noticing that $C_{i j k}=0$ by assumption, it follows that

$$
\sum_{j=1}^{n}\left|\nabla_{j} F\right|^{2}\left(\sum_{i \neq j, k \neq j}\left(\lambda_{i}-\lambda_{k}\right)^{2}\right)=0 .
$$

Since $c$ is a regular value of $F$ and $p \in \Sigma_{c}, \nabla F(p) \neq 0$. On the other hand, from (3.4) it is easy to see that if $\nabla F(p)$ has two or more nonzero components with respect to $\left\{E_{i}\right\}_{i=1}^{n}$, then $\lambda_{1}=\cdots=\lambda_{n}$, so the Ricci tensor has a unique eigenvalue. Otherwise, taking $\nabla_{1} F \neq 0$ and $\nabla_{i} F=0$ for $i=2, \cdots, n$, then $\nabla F=|\nabla F| E_{1}$, with $E_{1}=\nabla F /|\nabla F|$, is an eigenvector of $R c$, and $\lambda_{2}=\cdots=\lambda_{n}$. In either case, we conclude that $\nabla F$ is an eigenvector, and the Ricci tensor has the desired properties.

Remark 3.1. Fernández-López and García-Río [10] showed, by a different argument, that for shrinking solitons with harmonic Weyl tensor, the gradient of any potential function is an eigenvector of the Ricci tensor. 
Finally we show that the eigenvalues of the Ricci tensor and the scalar curvature $R$ are constant on level surfaces $\Sigma_{c}=\{F=c\}$.

Lemma 3.3. Let $c$ be a regular value of $F$ and $\Sigma_{c}=\{F=c\}$. Then:

(a) The function $G=|\nabla F|^{2}$ is constant on $\Sigma_{c}$; i.e. $G$ is a function of $F$ only.

(b) The scalar curvature $R$ of $\left(M^{n}, g_{i j}, F\right)$ is constant on $\Sigma_{c}$.

(c) The second fundamental form $h_{a b}$ of $\Sigma_{c}$ is of the form $h_{a b}=\frac{H}{n-1} g_{a b}$.

(d) The mean curvature $H$ is constant on $\Sigma_{c}$.

(e) $\Sigma_{c}$, with the induced metric $g_{a b}$, is of constant sectional curvature.

Proof. Let $\left\{e_{1}, e_{2}, \cdots, e_{n}\right\}$ be an orthonormal frame with $e_{1}=\nabla F /|\nabla F|$ and $e_{2}, \cdots, e_{n}$ tangent to $\Sigma_{c}$.

First of all, by (3.3) and Lemma 3.1 (ii),

$$
\nabla_{a} G=2 R c\left(\nabla F, e_{a}\right)=0, a=2, \cdots, n .
$$

Hence $G$ is constant on $\Sigma_{c}$, and so is $R$ by (2.3). This proves (a) and (b).

Next, since $R_{a b}=R_{a a} g_{a b}$ and $R_{22}=\cdots=R_{n n}$ by Lemma 3.1, we have

$$
h_{a b}=G^{-1 / 2} R_{a b}=\frac{H}{n-1} g_{a b}, a, b=2, \cdots, n,
$$

where

$$
H=G^{-1 / 2}\left(R-R_{11}\right) .
$$

Moreover, the Codazzi equation says that, for $a, b, c=2, \cdots, n$,

$$
R_{1 c a b}=\nabla_{a}^{\Sigma_{c}} h_{b c}-\nabla_{b}^{\Sigma_{c}} h_{a c} .
$$

Tracing over $b$ and $c$, we obtain

$$
0=R_{1 a}=\nabla_{a}^{\Sigma_{c}} H-\nabla_{b}^{\Sigma_{c}} h_{a b}=\left(1-\frac{1}{n-1}\right) e_{a} H
$$

proving (c) and (d).

Finally, by the Gauss equation, the sectional curvatures of $\left(\Sigma_{c}, g_{a b}\right)$ are given by

$$
R_{a b a b}^{\Sigma_{c}}=R_{a b a b}+h_{a a} h_{b b}-h_{a b}^{2}=R_{a b a b}+\frac{H^{2}}{(n-1)^{2}} .
$$

On the other hand, since $W_{i j k l}=0$,

$$
\begin{aligned}
R_{a b a b} & =\frac{2}{n-2} R_{a a}-\frac{R}{(n-1)(n-2)} \\
& =\frac{2 G^{1 / 2} H-R}{(n-1)(n-2)} .
\end{aligned}
$$

But we already showed that $G, H, R$ are constant on $\Sigma_{c}$. Therefore $\left(\Sigma_{c}, g_{a b}\right)$ has constant sectional curvature

$$
K_{c}=\frac{2 G^{1 / 2} H-R}{(n-1)(n-2)}+\frac{H^{2}}{(n-1)^{2}},
$$

proving (e).

With Lemma 3.3 in our hands, we now conclude the proof of Proposition 3.1. Recall that in any neighborhood, where $G \neq 0$, of the equipotential hypersurface

$$
\Sigma_{c}=:\{x \in M: F(x)=c\}
$$


of a regular value $c$ of $F$, we can express the metric $d s^{2}=g_{i j}(x) d x^{i} d x^{j}$ as

$$
d s^{2}=G^{-1}(F, \theta) d F^{2}+g_{a b}(F, \theta) d \theta^{a} d \theta^{b},
$$

where $\theta=\left(\theta^{2}, \cdots, \theta^{n}\right)$ denotes the intrinsic coordinates for $\Sigma_{c}$. Then Lemma 3.3 tells us that $G=G(F), g_{a b}=g_{a b}(F)$, and that $\left(\Sigma_{c}, g_{a b}\right)$ is a space form, with positive curvature (this follows from $(3.5)$ and the assumption that $\left(M^{n}, g_{i j}, F\right)$ has positive sectional curvature). Also, $F$ has exactly one minimum at some origin $x_{0}$; otherwise $\left(M^{n}, g_{i j}, F\right)$ would split out a flat factor, which is impossible. Hence, on $M \backslash\left\{x_{0}\right\}$ we have

$$
d s^{2}=G^{-1}(F) d F^{2}+\varphi^{2}(F) \bar{g},
$$

where $\bar{g}$ denotes the standard metric on the unit sphere $\mathbb{S}^{n-1}$, and $\phi$ is some smooth function on $M^{n}$ depending only on $F$ and vanishing only at $x_{0}$. Thus, $\left(M^{n}, g_{i j}, F\right)$ is a rotationally symmetric gradient steady soliton on $\mathbb{R}^{n}$. Therefore, it is the Bryant soliton.

Proof of Theorem 1.2. Now, by assumption, $\left(M^{n}, g_{i j}, F\right)$ is a complete noncompact locally conformally flat gradient steady soliton. By Proposition 2.5, we know that $\left(M^{n}, g_{i j}, F\right)$ has bounded and nonnegative curvature operator $0 \leq R m \leq C$. From Hamilton's strong maximum principle (see [13] and [15]), $\left(M^{n}, g_{i j}, F\right.$ ) is either of positive curvature operator, or its holonomy group reduces.

If $\left(M^{n}, g_{i j}, F\right)$ has positive curvature operator $R m>0$, then by Proposition 3.1/Theorem 1.1, $\left(M^{n}, g_{i j}, F\right)$ must be the Bryant soliton.

On the other hand, if $\left(M^{n}, g_{i j}, F\right)$ has reduced holonomy, then $\left(M^{n}, g_{i j}, F\right)$ is either a Riemannian product, or a locally symmetric space, or irreducible but not locally symmetric.

- Case (a): $\left(M^{n}, g_{i j}, F\right)$ is a Riemannian product.

It is known (cf. p.61 in [1]) that the only conformally flat Riemannian products are either the product of a space form $N^{n-1}$ with $\mathbb{S}^{1}$ or $\mathbb{R}^{1}$, or the product of two Riemannian manifolds, one with constant sectional curvature -1 and the other with constant sectional curvature 1 . However, since our $\left(M^{n}, g_{i j}\right)$ is a steady Ricci soliton with nonnegative sectional curvature, this implies that the latter case cannot occur and that in the former case $N^{n-1}$ must be flat. Hence $\left(M^{n}, g_{i j}, F\right)$ is flat in case (a).

- Case (b): $\left(M^{n}, g_{i j}, F\right)$ is a locally symmetric space.

In case $(\mathrm{b}),\left(M^{n}, g_{i j}, F\right)$ is necessarily Einstein. However, $\left(M^{n}, g_{i j}, F\right)$ is noncompact and of nonnegative sectional curvature. Therefore it follows that it must be Ricci flat and hence flat.

- Case (c): $\left(M^{n}, g_{i j}, F\right)$ is irreducible and not locally symmetric.

In this case the flatness of $\left(M^{n}, g_{i j}, F\right)$ follows from the holonomy classification theorem of Berger and Simons (cf. [1]), the fact that

$$
\left|W_{i j k l}\right|^{2} \geq \frac{3(m-1)}{m(m+1)(2 m-1)} R^{2}
$$

for Kähler manifolds of complex dimension $m>1$ (cf. Proposition 2.68 in [1]), and that our $\left(M^{n}, g_{i j}, F\right)$ is noncompact, conformally flat and of nonnegative sectional curvature.

Thus we have shown that in all of the above three cases, $\left(M^{n}, g_{i j}, F\right)$ are flat. This completes the proof of Theorem 1.2. 
Remark 3.2. Shortly after our work appeared on the arXiv, Catino and Mantegazza [6] obtained a similar result to our Theorem 1.1 for dimension $n \geq 4$ by studying the evolution of the Weyl tensor under the Ricci flow. However, as pointed out in [6], their argument does not work for $n=3$.

\section{FURTHER REMARKS}

In this section, we point out that several lemmas in the proof of Theorem 1.1 for gradient steady Ricci solitons also hold for gradient shrinking and expanding Ricci solitons satisfying

$$
R_{i j}=\nabla_{i} \nabla_{j} F+\rho g_{i j}
$$

with $\rho=1 / 2$ for shrinkers and $\rho=-1 / 2$ for expanders, respectively. In particular, our method, when combined with a result of Kotschwar [18, yields another proof of the classification theorem for $n$-dimensional $(n \geq 4)$ complete locally conformally flat shrinking gradient solitons (see Proposition 4.1).

Remark 4.1. A similar version of Lemma 3.1 is valid for locally conformally flat gradient shrinkers and expanders.

First of all, similar arguments to those in Lemma 2.1 imply that

$$
\nabla_{i} R=-2 R_{i j} \nabla_{j} F
$$

and

$$
R+|\nabla F|^{2}+2 \rho F=C_{0}
$$

for some constant $C_{0}$. Hence, by defining

$$
G_{\rho}=: G+2 \rho F=|\nabla F|^{2}+2 \rho F,
$$

we have

$$
R+G_{\rho}=C_{0}
$$

and

$$
\nabla G_{\rho}=-\nabla R=2 R c(\nabla F, \cdot) .
$$

Therefore, by replacing $G=|\nabla F|^{2}$ by $G_{\rho}=G+2 \rho F$ and carrying out the same arguments as in the proof of Lemma 3.1, we obtain

Lemma 4.1. For any $n$-dimensional gradient shrinking or expanding soliton ( $n \geq$ 3 ) with vanishing Weyl tensor $W_{i j k l}=0$, the Cotton tensor $C_{i j k}$ has the property that

$$
\left|C_{i j k}\right|^{2}=\frac{1}{(n-2)^{2}}\left(\left|R_{i k} \nabla_{j} F-R_{i j} \nabla_{k} F\right|^{2}-\frac{2}{(n-1)}\left|R \nabla F-\frac{1}{2} \nabla G_{\rho}\right|^{2}\right) .
$$

Consequently, Lemma 3.2 and Lemma 3.3 hold for locally conformally flat gradient shrinkers and expanders as well.

Lemma 4.2. Let $\left(M^{n}, g_{i j}, F\right)$ be any $n$-dimensional, $n \geq 3$, complete locally conformally flat gradient shrinking or expanding Ricci soliton satisfying (4.1). Then, at any regular point $p \in \Sigma_{c}$, the Ricci tensor of $\left(M^{n}, g_{i j}, F\right)$ either has a unique eigenvalue $\lambda$ or has two distinct eigenvalues $\lambda$ and $\mu$ of multiplicity 1 and $n-1$, respectively. In either case, $e_{1}=\nabla F /|\nabla F|$ is an eigenvector with eigenvalue $\lambda$. In other words, for any orthonormal basis $e_{2}, \cdots, e_{n}$ tangent to the level surface $\Sigma_{c}$ at 
$p$, the Ricci tensor has the following properties:

(i) $R c\left(e_{1}, e_{1}\right)=R_{11}$,

(ii) $R c\left(e_{1}, e_{b}\right)=R_{1 b}=0, b=2, \cdots, n$,

(iii) $R c\left(e_{a}, e_{b}\right)=R_{a a} \delta_{a b}, a, b=2, \cdots, n$,

where either $R_{11}=\cdots=R_{n n}=\lambda$ or $R_{11}=\lambda$ and $R_{22}=\cdots=R_{n n}=\mu$.

Lemma 4.3. Let $\left(M^{n}, g_{i j}, F\right)$ be any $n$-dimensional, $n \geq 3$, complete locally conformally flat gradient shrinking or expanding Ricci soliton satisfying (4.1), and let $c$ be a regular value of $F$ and $\Sigma_{c}=\{F=c\}$. Then:

(a) The function $G=|\nabla F|^{2}$ is constant on $\Sigma_{c}$; i.e., $G$ is a function of $F$ only.

(b) $R$, the scalar curvature of $\left(M^{n}, g_{i j}, F\right)$, is constant on $\Sigma_{c}$.

(c) The second fundamental form $h_{a b}$ of $\Sigma_{c}$ is of the form $h_{a b}=\frac{H}{n-1} g_{a b}$.

(d) The mean curvature $H$ is constant on $\Sigma_{c}$.

(e) $\left(\Sigma_{c}, g_{a b}\right)$ is of constant sectional curvature $K_{c, \rho}$.

Note that for shrinking solitons, we have $K_{c, 1 / 2} \geq 0$, according to [23].

Remark 4.2. There is another formula for $\left|C_{i j k}\right|^{2}$ which is valid for all $n$-dimensional $(n \geq 3)$ complete gradient shrinking, steady, and expanding Ricci solitons with vanishing Weyl tensor. This new formula relates $\left|C_{i j k}\right|^{2}$ more explicitly to the geometry of the level surfaces of $F$ and immediately implies Lemma 3.2/4.2 and Lemma 3.3/4.3 (a)-(d).

Lemma 4.4. For any n-dimensional gradient shrinking, or steady, or expanding soliton $\left(M^{n}, g_{i j}, F\right)(n \geq 3)$ with vanishing Weyl tensor $W_{i j k l}=0$, we have

$$
\left|C_{i j k}\right|^{2}=\frac{2 G^{2}}{(n-2)^{2}}\left|h_{a b}-\frac{H}{n-1} g_{a b}\right|^{2}+\frac{1}{2(n-1)(n-2)}\left|\nabla_{a} G\right|^{2},
$$

where $h_{a b}$ and $H$ are, as in Lemma 3.3, the second fundamental form and the mean curvature for the level surface $\Sigma_{c}=\{F=c\}$ at any regular value $c$ of $F$.

Proof. Denote again

$$
G=|\nabla F|^{2} \quad \text { and } \quad G_{\rho}=G+2 \rho F
$$

so that $G_{0}=G$. Let $c$ be any regular value of the potential function $F$ and consider the corresponding equipotential hypersurface

$$
\Sigma_{c}=:\{x \in M: F(x)=c\} .
$$

Let $\left\{e_{1}, e_{2}, \cdots, e_{n}\right\}$ be any orthonormal frame, with $e_{1}=\nabla F /|\nabla F|=\nabla F / \sqrt{G}$ and $e_{2}, \cdots, e_{n}$ tangent to $\Sigma_{c}$. Then the second fundamental form $h_{a b}$ and the mean curvature $H$ are given respectively by

$$
\begin{aligned}
h_{a b} & =\left\langle\nabla_{a} e_{1}, e_{b}\right\rangle=\left\langle\nabla_{a} \frac{\nabla F}{\sqrt{G}}, e_{b}\right\rangle \\
& =\frac{1}{\sqrt{G}} R_{a b}, \quad a, b=2, \cdots, n
\end{aligned}
$$

and

By (4.2),

$$
H=\frac{1}{\sqrt{G}}\left(R-R_{11}\right)
$$

$$
R_{11}=\frac{1}{G} R c(\nabla F, \nabla F)=\frac{1}{2 G} \nabla F \cdot \nabla G_{\rho}
$$


and

Moreover,

$$
R_{1 a}=\frac{1}{\sqrt{G}} R c\left(\nabla F, e_{a}\right)=\frac{1}{2 \sqrt{G}} \nabla_{a} G_{\rho} .
$$

$$
\begin{aligned}
\left|\nabla_{\Sigma_{c}} G_{\rho}\right|^{2} & =\sum_{a=2}^{n}\left|\nabla_{a} G_{\rho}\right|^{2} \\
& =\left|\nabla G_{\rho}\right|^{2}-\frac{1}{G}\left|\nabla G_{\rho} \cdot \nabla F\right|^{2} .
\end{aligned}
$$

Thus, by direct computations, we obtain

$$
\begin{aligned}
\left|h_{a b}-\frac{H}{n-1} g_{a b}\right|^{2}= & \left|h_{a b}\right|^{2}-\frac{H^{2}}{n-1} \\
= & \frac{1}{G}\left(|R c|^{2}-2 \sum_{a=2}^{n} R_{1 a}^{2}-R_{11}^{2}\right)-\frac{\left(R-R_{11}\right)^{2}}{(n-1) G} \\
= & \frac{1}{G}|R c|^{2}+\frac{R}{(n-1) G^{2}} \nabla F \cdot \nabla G_{\rho}-\frac{1}{2 G^{2}}\left|\nabla G_{\rho}\right|^{2} \\
& +\frac{n-2}{4(n-1) G^{3}}\left|\nabla F \cdot \nabla G_{\rho}\right|^{2}-\frac{R^{2}}{(n-1) G} .
\end{aligned}
$$

Now noticing that, by Lemma 4.1, we also have

$$
\begin{aligned}
\left|C_{i j k}\right|^{2}= & \frac{2 G}{(n-2)^{2}}|R c|^{2}-\frac{1}{2(n-2)^{2}}\left|\nabla G_{\rho}\right|^{2} \\
& -\frac{1}{2(n-1)(n-2)^{2}}\left(\left|\nabla G_{\rho}\right|^{2}-4 R \nabla G_{\rho} \cdot \nabla F+4 R^{2} G\right),
\end{aligned}
$$

one can therefore verify directly that

$$
\frac{2 G^{2}}{(n-2)^{2}}\left|h_{a b}-\frac{H}{n-1} g_{a b}\right|^{2}=\left|C_{i j k}\right|^{2}-\frac{1}{2(n-1)(n-2)}\left|\nabla_{\Sigma_{c}} G_{\rho}\right|^{2} .
$$

This completes the proof of Lemma 4.4.

Remark 4.3. Based on Lemma 4.3 for shrinkers, it is not hard to see that the universal cover $\left(\tilde{M}^{n}, \tilde{g}_{i j}, \tilde{F}\right)$ of a complete locally conformally flat gradient shrinking Ricci soliton is a rotationally symmetric gradient shrinking Ricci soliton and $\tilde{M}^{n}$ is diffeomorphic to either $\mathbb{R}^{n}$, or $\mathbb{S}^{n}$, or $\mathbb{S}^{n-1} \times \mathbb{R}$. Combining this with the result of Kotschwar 18, one obtains another proof of the following classification theorem for $n$-dimensional $(n \geq 4)$ complete locally conformally flat shrinking gradient solitons (which is first due to the combined works of Z.-H. Zhang and Ni-Wallach, and also the combination of more recent work of Munteanu-Sesum and the earlier works of either Petersen-Wylie, or X. Cao, B. Wang and Z. Zhang).

Proposition 4.1. Any $n$-dimensional, $n \geq 4$, complete locally conformally flat gradient shrinking Ricci soliton is a finite quotient of $\mathbb{R}^{n}$, or $\mathbb{S}^{n}$, or $\mathbb{S}^{n-1} \times \mathbb{R}$.

\section{ACKNowledgments}

The first author is grateful to Rick Schoen for bringing the papers [16, 21] to his attention. He also would like to thank Bing-Long Chen and Xi-Ping Zhu for helpful discussions, and S.-C. Chu for pointing out the reference [12]. 


\section{REFERENCES}

[1] Besse, A., Einstein Manifolds. Springer-Verlag, Berlin, 1987. MR0867684 (88f:53087)

[2] Bryant, R., unpublished work.

[3] Cao, H.-D., Existence of gradient Kähler-Ricci solitons, Elliptic and Parabolic Methods in Geometry (Minneapolis, MN, 1994), A K Peters, Wellesley, MA (1996), 1-16. MR1417944 (98a:53058)

[4] Cao, H.-D., Recent progress on Ricci solitons, Recent advances in geometric analysis, 1-38, Adv. Lect. Math. (ALM) 11, Int. Press, Somerville, MA, 2010. MR2648937

[5] Cao, H.-D. and Zhu, X.-P., A complete proof of the Poincaré and geometrization conjectures - application of the Hamilton-Perelman theory of the Ricci flow, Asian J. Math. 10 (2006), no. 2, 165-492. MR.2233789 (2008d:53090)

[6] Catino, G. and Mantegazza, C., Evolution of the Weyl tensor under the Ricci flow, arXiv: 0910.4761 .

[7] Chen, B.-L., Strong uniqueness of the Ricci flow, J. Differential Geom. 82 (2009), 363-382. MR2520796 (2010h:53095)

[8] Chow, B. et al., The Ricci flow: techniques and applications. Part I. Geometric aspects. Mathematical Surveys and Monographs, 135. American Mathematical Society, Providence, RI, 2007. MR2302600 (2008f:53088)

[9] Chu, S.-C., Geometry of 3-dimensional gradient Ricci solitons with positive curvature, Comm. Anal. Geom., 13 (2005), 129-150. MR2154669 (2006h:53060)

[10] Fernández-López, M. and García-Río, E., Rigidity of shrinking Ricci solitons, preprint (2009).

[11] Gromoll, D. and Meyer, W., On complete open manifolds of positive curvature, Ann. of Math. (2) 90 (1969), 75-90. MR0247590 (40:854)

[12] Guo, H., Area growth rate of the level surface of the potential function on the 3-dimensional steady Ricci soliton, Proc. Amer. Math. Soc. 137 (2009), no. 6, 2093-2097. MR2480291 (2010i:53123)

[13] Hamilton, R. S., Four-manifolds with positive curvature operator, J. Differential Geom. 24 (1986), 153-179. MR0862046 (87m:53055)

[14] Hamilton, R. S., The Ricci flow on surfaces, Contemporary Mathematics 71 (1988), 237-261. MR.0954419 (89i:53029)

[15] Hamilton, R. S., The formation of singularities in the Ricci flow, Surveys in Differential Geometry (Cambridge, MA, 1993), 2, 7-136, International Press, Cambridge, MA, 1995. MR:1375255 (97e:53075)

[16] Israel, W., Event horizons in static vacuum space-times, Phys. Rev., 164, no. 5 (1967), 1776-1779.

[17] Ivey, T., Ricci solitons on compact three-manifolds, Diff. Geom. Appl. 3 (1993), 301-307. MR.1249376 (94j:53048)

[18] Kotschwar, B., On rotationally invariant shrinking Ricci solitons, Pacific J. Math. 236 (2008), 73-88. MR2398988 (2009k:53171)

[19] Munteanu, O. and Sesum, N., On gradient Ricci solitons, arXiv:0910.1105.

[20] Perelman, G., The entropy formula for the Ricci flow and its geometric applications, arXiv:math.DG/0211159.

[21] Robinson, D. C., A simple proof of the generalization of Israel's theorem, General Relativity and Gravitation, 8, no. 8 (1977), 695-698.

[22] Zhang, Z.-H., On the completeness of gradient Ricci solitons, Proc. Amer. Math. Soc. 137 (2009), no. 8, 2755-2759. MR2497489 (2010a:53057)

[23] Zhang, Z.-H., Gradient shrinking solitons with vanishing Weyl tensor, Pacific J. Math. 242 (2009), no. 1, 189-200. MR2525510(2010f:53116)

Department of Mathematics, Lehigh University, Bethlehem, Pennsylvania 18015

E-mail address: huc2@lehigh.edu

Department of Mathematics, Lehigh University, Bethlehem, Pennsylvania 18015

E-mail address: qic208@lehigh.edu 